\section{Electrophoresis of Unlabeled Proteins in a Sequencing Gel Apparatus}

BioTechniques 28:36-38 (January 2000)

In an earlier issue of this journal, Whelan and Taylor (4) described the adaptation of the Sequi-Gen ${ }^{\circledR}$ electrophoretic cell (Bio-Rad Laboratories, Hercules, CA, USA) for analysis of proteins. Their paper demonstrated that, with slight modifications of the plate assembly and gel loading protocols, it was possible to obtain a large degree of separation of the radiolabeled polypeptides electrophoresed, as checked after gel drying onto chromatography paper and autoradiography. In the same paper, the authors stated that a full-length run of unlabeled proteins was not recommended for the handling of long floating gels during Coomassie Blue or silver staining and suggested electrophoresis of the samples for half of the length of the gel, followed by cutting the latter for stain processing. Here, we produce evidence that unlabeled protein mixtures, such as cell protein extracts, can be successfully submitted to full-length electrophoresis in sequencing gels without significant modifications of the preparation technique as described by the manufacturers.

MDA-MB231 breast cancer cells were maintained in culture in RPMI 1640 medium plus $10 \%$ fetal calf serum for 5 days and total proteins were obtained as reported by Wang et al. (3). Essentially, subconfluent cells were washed $3 \times$ with prewarmed PBS, lysed in a flask with $20 \mathrm{mmol} / \mathrm{L}$ Tris$\mathrm{HCl}, \mathrm{pH} 7.4$, containing 2\% SDS and the mixture of protease inhibitors recommended. The extract was precipitated with $100 \%$ trichloroacetic acid (TCA), washed with $2.5 \%$ TCA, neutralized with $3 \mathrm{~mol} / \mathrm{L}$ Tris-base and stored at $-20^{\circ} \mathrm{C}$ in $1.5 \mathrm{~mol} / \mathrm{L}$ Tris-base until used. The protein concentration was evaluated by the Lowry method.

To prepare the electrophoretic apparatus, the reservoir-bearing plate of a Sequi-Gen cell $(21 \times 40 \mathrm{~cm})$ was treated with $500 \mu \mathrm{L}$ of Silanization Solution I (Fluka, Buchs, Switzerland) or SigmaCote $^{\circledR}$ (Sigma, St. Louis, MO, USA) to avoid gel attachment. The glass plate was treated with a freshly prepared binding solution $(5 \mu \mathrm{L} \gamma$-methacryloxypropyltrimethoxysilane from Sigma and $5 \mu \mathrm{L}$ glacial acetic acid in $1 \mathrm{~mL} \mathrm{95 \%}$ ethanol) to cross-link the gel. The glassplate sandwich was then assembled with the standard $0.4 \mathrm{~mm}$ thick spacers.

A hundred milliliters of SDS-polyacrylamide gel electrophoresis solution were prepared according to standard protocols (1) and degassed. Five milliliters of this solution were mixed with $50 \mu \mathrm{L}$ of $10 \%$ ammonium persulfate and $5 \mu \mathrm{L}$ of TEMED and used for gel bottom sealing according to the manufacturers' instructions; after successful polymerization of the seal, the separating gel was poured slowly with a 50 $\mathrm{mL}$ syringe, and the comb was inserted before the gel polymerized.

We have successfully prepared both monophasic slabs consisting of a uniform separating gel and biphasic slabs containing a stacking gel with no remarkable difference in protein band resolution. However, in the latter case, the gel comb should be inserted more deeply between the plates to allow space $(\sim 0.5 \mathrm{~cm})$ for the polymerization of a stacking gel mixture, prepared according to the standard protocol (1). Thus, after setting of the separating gel and comb removal, the gel surface was washed with distilled water that was blotted onto a piece of filter paper, the stacking gel solution poured and the comb inserted again before the polymerization of the upper gel. Following this protocol, one complete gel was produced in approximately $2 \mathrm{~h}$.

After assembling the apparatus, pouring the running buffer and flushing the wells with a $5 \mathrm{~mL}$ syringe, the protein samples were mixed at a 1:1 ratio with a $2 \times$ concentrated dithiothreitolcontaining sample buffer, boiled for 3 min, and up to $6 \mu \mathrm{L}$ of the mixture was loaded into each well and electrophoresed for $14 \mathrm{~h}$ at $300 \mathrm{~V}$. At the end of the run, the electrophoretic cell was disassembled, and the gel, attached to the glass plate, was easily handled for staining into containers of the appropriate size, such as the $48 \times 27 \mathrm{~cm}$ polypropylene trays from Aldrich Chemical (Milwaukee, WI, USA). The gel was silver-stained according to the protocol published by Swain and Ross (2).
The water used for all the solutions and washings was Milli- ${ }^{\circledR}$ (Millipore, Bedford, MA, USA) grade. Accordingly, the gel was fixed with $40 \%$ ethanol/ $10 \%$ acetic acid for $10 \mathrm{~min}$, rinsed with water for $10 \mathrm{~min}$ and fixed/sensitized with $0.05 \%$ glutaraldehyde $/ 0.01 \%$ formalin/40\% ethanol for $5 \mathrm{~min}$. After sequential rinsing with $40 \%$ ethanol and water for $20 \mathrm{~min}$ each, the gel was sensitized with $0.2 \mathrm{~g}$ sodium thiosulphate/L for $1 \mathrm{~min}$, rinsed with water for $2 \times 1$ min and treated with $0.1 \%$ silver nitrate for $20 \mathrm{~min}$. After rinsing with water for $1 \mathrm{~min}$, the stain was developed with $2.5 \%$ sodium carbonate $/ 0.04 \%$ formalin; it is suggested to incubate the gel with an aliquot of the developing solution until the latter turns yellow and then substituting with a fresh one to avoid silver deposition onto the gel surface and to improve the quality of the stain.

When the desired stain intensity was

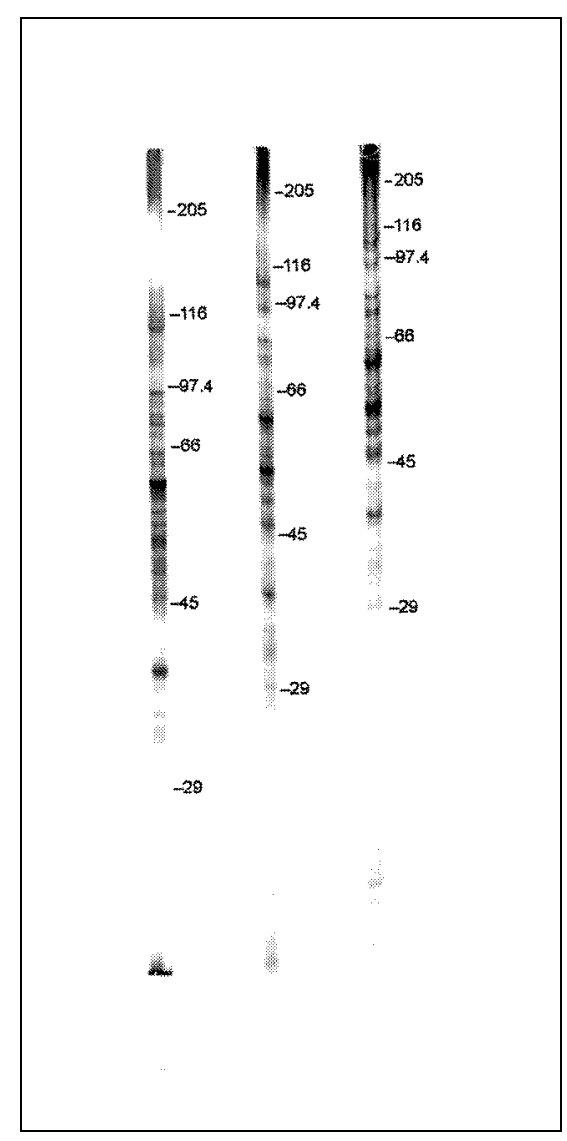

Figure 1. Panel of full-length $8 \%$ (left), $10 \%$ (center) and $12 \%$ (right) Polyacrylamide gels of protein extracts from MDA-MB231 cells run in parallel with a high molecular weightstandard protein mixture (Sigma). Molecular masses are indicated on the right in kilodaltons. 
attained, the development was stopped with $5 \%$ acetic acid and, after $10 \mathrm{~min}$, the gel was finally washed in distilled water and air-dried onto the glass support. Air-drying allows the long and thin gel to be easily submitted to photographic and/or digital recording using a sequencing gel-sized scanner, such as the HP ScanJet 4C/T (Hewlett-Packard, Palo Alto, CA, USA). This avoids wethandling or the need of an apparatus for gel-drying between cellophane sheets. Once examined and/or recorded, the dried gel can be detached from the glass plate after overnight rinsing with warm $5 \mathrm{~mol} / \mathrm{L} \mathrm{NaOH}$ and the latter reused after extensive washing with detergent, ethanol and distilled water.

In our laboratory, we have applied the full-length electrophoresis technique several times to the study of the pattern of proteins synthesized by MDA-MB231 tumor cells in response to microenvironmental stimuli (data not reported). Figure 1 shows that the protein gels submitted to full-length electrophoresis exhibit a sensitive staining of protein bands (from 30-60, depending on acrylamide concentration) and a low level of background, which make them suitable for documentation and for software-assisted qualitative and quantitative analysis of the electrophoretic pattern. The number of resolved proteins cannot be compared to that obtained by 2-D electrophoresis (see the 2-D protein map from MDA-MB231 cells available online at http://www.anl.gov/BIO/PMG/ projects/index_hbreast.html). Nevertheless, full-length 1-D electrophoresis is a faster method that $(i)$ does not require special consumables and expensive apparatus (such as ampholytes and IPG gel systems), (ii) improves the resolution of the pattern of polypeptides submitted to monodimensional electrophoresis and (iii) expands the applications of the DNA sequencing apparatus for protein studies.

\section{REFERENCES}

1.Sambrook, J., E.F. Fritsch and T. Maniatis. 1989. Molecular cloning: A Laboratory manual, 2nd ed. CSH Laboratory Press, Cold Spring Harbor, NY.

2.Swain, M. and N.W. Ross. 1995. A silver stain protocol for proteins yielding high reso- lution and transparent background in sodium dodecyl sulfate-polyacrylamide gels. Electrophoresis 16:948-951.

3.Wang, K.K.W., A. Posner and I. Hajimohammadreza. 1996. Total protein extraction from cultured cells for use in electrophoresis and western blotting. BioTechniques 20:662668.

4.Whelan, K.F. and D.E. Taylor. 1996. Use of a DNA sequencing gel apparatus for analysis of polypeptides. BioTechniques 21:805-808.

This study was supported by MURST (R.S. ex 60\%). Address correspondence to Dr. Claudio Luparello, Dipartimento di Biologia Cellulare e dello Sviluppo, Viale delle Scienze, 90128 Palermo, Italy. Internet: clupar@tin.it

Received 9 July 1999; accepted 29 September 1999.

Claudio Luparello

University of Palermo

Palermo, Italy

\section{Rapid Histone Extraction for Electrophoretic Analysis}

\author{
BioTechniques 28:38-46 (January 2000)
}

Histones, the class of basic proteins that complex with DNA to form chromatin (11), consist of multiple posttranslationally modified forms and isoprotein species (13). Several of these have recently been specifically implicated in such cellular functions as transcription (2), detection of DNA damage $(7,8)$ and mitosis $(12)$, as well as a human genetic disease, Coffin-Lowry syndrome (9). While the major histone protein families can usually be resolved on SDS gels, these gels are not capable of resolving either the histone isoprotein species or the post-translationally modified forms. On the other hand, much of the complexity of histone species and forms can be resolved on acidic gels with or without Triton ${ }^{\circledR} \mathrm{X}$ $100(1,6,14)$. However, these gels have not been as widely used as the SDS gels because sample preparation is more laborious, often requiring dialysis or precipitation steps that can lead to selective loss, degradation and oxidation of histone forms.

Histones can be extracted in 0.5 $\mathrm{mol} / \mathrm{L} \mathrm{HCl}$ or $0.25 \mathrm{~mol} / \mathrm{L} \mathrm{H}_{2} \mathrm{SO}_{4}$ solutions and analyzed on gels containing acetic acid. Bonner et al. (1) had shown that it is possible to neutralize the extraction acid, add urea and acetic acid and, without further preparation, load the samples onto a type of acetic acid gel that incorporates a discontinuous buffer system. Discontinuous buffer systems permit the concentration of protein components from varied ionic solutions $(4,5)$. Because both the extraction and gel buffers for histones are acidic, we decided to examine whether these gel systems are compatible with a strongly acidic sample. If they were found to be so, then the histone extraction buffer could also serve as the gel loading buffer, thus greatly simplifying histone sample preparation. In this report, we describe procedures by which histone extracts of cells and tissues can be transferred directly from nuclear pellets onto these types of gels. In addi- 